Gynäkologische Endokrinologie 2013 · 11:89-94 DOI 10.1007/s10304-012-0517-3

Online publiziert: 12. April 2013

(c) Springer-Verlag Berlin Heidelberg 2013
A. Santi · S. Rohner

Kinderwunschzentrum, Universitätsklinik für Frauenheilkunde, Inselspital Bern, Bern

\title{
Anatomische Ursachen bei habituellen Aborten
}

\section{Diagnostik und Therapie}

Die Inzidenz uteriner Fehlbildungen ist bei Frauen mit habituellen Aborten im Vergleich zu normal fertilen Frauen signifikant erhöht (ca. 13\% vs. $4 \%$; • Tab. 1, [5]). Diese Häufigkeit steigt auf bis zu 25\% an, wenn Aborte ab der zehnten Schwangerschaftswoche (SSW) auftreten.

Zur invasiven Diagnostik wird insbesondere eine Kombination aus Laparoskopie (LSC) und Hysteroskopie (HSC) eingesetzt. Seit einigen Jahren spielen daneben auch Verfahren wie die Magnetresonanztomographie (MRT) und v. a. der 3-D-Ultraschall (3-D-US) eine zunehmend wichtige Rolle. Ziel dieses Beitrags ist eine Zusammenfassung der heutigen Evidenz mit Empfehlungen für eine rationale Diagnostik und Behandlung uteriner Missbildungen bei habituellen Aborten.

\section{Ätiologie der uterinen Fehlbildungen}

Fehlbildungen im weiblichen Genitale können in allen 4 Entwicklungsstadien der Müller-Gänge auftreten:

1. Kaudale Elongation: Eine gestörte Entwicklung in dieser ersten Phase führt zu einer kompletten Agenesie eines oder beider Müller-Gänge, wie beispielsweise bei einem Uterus unicornis.

2. Im nächsten Schritt fusionieren die 2 Gänge in der Mitte: eine gestörte Fusion hat eine inkomplette Verschmelzung der Müller-Gänge zur Folge, wie beim Uterus didelphys oder bicornis.

3. Danach beginnt etwa in der zehnten SSW die Kanalisierung: Zwei separate
Kanäle werden hergestellt und durch ein Septum getrennt. Die unzureichende Kanalisierung kann zu einem Uterus unicornis mit rudimentärem Horn (mit oder ohne Verbindung) führen.

4. Das Septum wird danach von kaudal nach kranial resorbiert (bis etwa zur 20. SSW): In dieser Phase entsteht das Cavum uteri. Störungen führen zu den am häufigsten diagnostizierten Fehlbildungen, dem Uterus septus und subseptus. Typisch für diese angeborenen Fehlbildungen sind Aborte im späten ersten Trimenon.

\section{Klassifikation}

Das Fehlen einer einheitlichen Klassifikation der uterinen Fehlbildungen ist ein seit Jahren bekanntes Problem. Die am häufigsten verwendete Klassifikation ist die Einteilung der American Society for Reproductive Medicine (ASRM; • Abb. 1, [2]). Der Vorteil dieser Klassifikation ist, dass praktisch alle uterinen Fehlbildungen mit diesem System beschrieben werden können. Nachteilig ist, dass die Zervix und die Tuben nicht berücksichtigt werden.

Kürzlich wurde eine neue europäische Klassifikation von der European Society of Human Reproduction and Embryology (ESHRE) und der European Society for Gynaecological Endoscopy (ESGE) vorgeschlagen (• Abb. 2, [11]): Ziel ist eine Vereinfachung der Subklassifikationen und gleichzeitig eine Zuteilung abhängig von der embryologischen Entwicklung. Es wurde versucht, die positiven Aspekte der international gut akzeptierten und weitläufig verwendeten ASRM-Einteilung beizubehalten, mit der Möglichkeit, später auch andere Anomalien, v. a. zervikale und vaginale, $\mathrm{zu}$ berücksichtigen.

\section{Diagnostik der Fehlbildung}

In den vergangenen 10 Jahren wurde mithilfe verschiedener Studien versucht, eine rationale Diagnostik der uterinen Fehlbildungen bei habituellen Aborten zu definieren [16]. Die Hysterosalpingographie (HSG) wird nach wie vor häufig eingesetzt, obwohl ihre diagnostische Sicherheit stark limitiert ist, insbesondere wenn nicht auch ein Gynäkologe an der Beurteilung der Uterusanatomie beteiligt ist [17]. Die HSG findet $z$. T. noch als Routinediagnostik bei Frauen mit habituellen Aborten Anwendung, was aber nicht mehr medizinisch angezeigt ist.

Die 2-D-Sonographie ist heutzutage immer die erste diagnostische Stufe: Schnell, günstig und praktisch ohne Risiken für die Patientin erlaubt sie eine sichere Diagnostik. Limitierender Faktor ist v. a. die Beurteilung des Cavum uteri, sodass sich in den letzten Jahren der immer häufiger durchgeführte 3-D-US zur Methode der Wahl entwickelt hat. Zahlreiche Arbeiten belegen seine Genauigkeit [6]. Sowohl die äußere als auch die innere Kontur des Uterus können gut dargestellt werden. Es wird empfohlen, die Sonographie in der zweiten Zyklushälfte durchzuführen. Der 3-D-US ist unkompliziert, schnell und deutlich günstiger als eine MRT oder ein invasiver Eingriff. Auch die Sonohysterographie - die Ultra- 


\begin{tabular}{|c|c|c|c|c|c|c|c|c|c|}
\hline Population & $\begin{array}{l}\text { Untersu- } \\
\text { chung }\end{array}$ & $\begin{array}{l}\text { Fallzahl Pa- } \\
\text { tientinnen }\end{array}$ & $\begin{array}{l}\text { Prävalenz } \\
\text { aller Fehlbil- } \\
\text { dungen (\%) }\end{array}$ & $\begin{array}{l}\text { Uterus } \\
\text { arcuatus } \\
(\%)\end{array}$ & $\begin{array}{l}\text { Uterus sep- } \\
\text { tus/subsep- } \\
\text { tus (\%) }\end{array}$ & $\begin{array}{l}\text { Uterus } \\
\text { bicornis } \\
(\%)\end{array}$ & $\begin{array}{l}\text { Uterus } \\
\text { unicornis } \\
(\%)\end{array}$ & $\begin{array}{l}\text { Uterus } \\
\text { didelphys } \\
(\%)\end{array}$ & $\begin{array}{l}\text { Andere } \\
\text { Fehlbild- } \\
\text { ungen (\%) }\end{array}$ \\
\hline \multirow{2}{*}{ Allgemein } & Optimal $^{\mathrm{a}}$ & 5163 & 5,5 & 3,9 & 2,0 & 0,4 & 0,1 & 0,3 & 0,1 \\
\hline & Suboptimal $^{b}$ & 52.590 & 4,0 & 2,0 & 0,2 & 0,2 & 0,2 & 0,1 & 2,5 \\
\hline \multirow{2}{*}{$\begin{array}{l}\text { Sterilitätspatien- } \\
\text { tinnen }\end{array}$} & Optimal $^{\mathrm{a}}$ & 10.303 & 8,0 & 1,8 & 3,0 & 1,0 & 0,5 & 0,3 & 0,9 \\
\hline & Suboptimal $^{b}$ & 8643 & 6,1 & 5,8 & 2,7 & 0,8 & 0,8 & 0,4 & 1,0 \\
\hline \multirow{2}{*}{$\begin{array}{l}\text { Patientinnen mit ha- } \\
\text { bituellen Aborten }\end{array}$} & Optimal $^{\mathrm{a}}$ & 2082 & 13,3 & 2,9 & 5,3 & 2,1 & 0,5 & 0,6 & 0,9 \\
\hline & Suboptimal $^{b}$ & 3961 & 15,8 & 8,9 & 4,3 & 2,8 & 0,5 & 0,6 & 4,5 \\
\hline
\end{tabular}

a Optimale Untersuchungstechnik zur Diagnostik gemäß [5]: 3-D-Sonographie, Laparoskopie + Hysteroskopie, Magnetresonanztomographie oder Sonohysterosalpingographie. ${ }^{b}$ Suboptimale Untersuchung [5]: 2-D-Sonographie, Hysteroskopie allein, Hysterosal pingographie, klinische Beurteilung bei einer Sectio caesarea.

Tab. 2 Die Sicherheit der diagnostischen Verfahren. (Modifiziert nach [18])

\begin{tabular}{|llll} 
Klasse & Genauigkeit der Untersuchung ${\left(\text {,accuracy }{ }^{\prime}\right)^{\text {a }}}^{\text {a }}$ & $\begin{array}{l}\text { Klassifikation eines } \\
\text { Subtyps möglich? }\end{array}$ & Verfahren \\
\hline la & $>90 \%$ & Ja & $\frac{3-D-U S}{\text { Sono-HSG }}$ \\
\cline { 3 - 4 } & & & LSC+HSC \\
\hline Ib & $>90 \%$ & Nein & HSC \\
\hline II & $<90 \%$ & Nein & 2-D-US \\
\hline III & Unklar & & HSG \\
\hline
\end{tabular}

a Definition in [18]: (Anzahl richtig positiv + richtig negativ)/(Anzahl richtig positiv + falsch positiv + falsch negativ + richtig negativ).

LSC Laparoskopie; HSC Hysteroskopie; HSG Hysterosalpingographie; MRT Magnetresonanztomographie; US Ultraschall.

schalluntersuchung mit flüssigkeitsgefülltem Cavum uteri - kann mit guten Resultaten eingesetzt werden, da nicht zuletzt die Beurteilung des Cavums im Vergleich zu einer routinemäßigen 2-D-Sonographie deutlich besser ist [10].

\section{I) Die sonographische Untersuchung sollte in der zweiten Zyklushälfte durchgeführt werden}

Die MRT ermöglicht eine sehr genaue Diagnostik, da die Differenzierung zwischen Myometrium und Endometrium sehr klar ist. Es ist deshalb auch möglich, funktionelle Endometriuminseln bei rudimentären Uteri zu diagnostizieren. Von Vorteil ist darüber hinaus die im Vergleich zur Sonographie bessere Darstellung der Zervix und der Vagina. Da die MRT aber sehr teuer und für die Patientin aufwendig ist, ist ihre Anwendung nur bei Verdacht auf komplizierte uterine Fehlbildungen angezeigt $[3,4]$.
Der Goldstandard bleibt die Durchführung einer Hysteroskopie (HSC), falls notwendig kombiniert mit einer LSC. Diese invasive Diagnostik sollte aber nicht routinemäßig bei allen Frauen mit habituellen Aborten eingesetzt werden, da die Risiken für die Patientinnen mit der Operation ansteigen, die Kosten deutlich höher sind und schließlich die definitive Diagnose der Fehlbildung immer durch die subjektive Beurteilung - und Erfahrung! - des Operateurs beeinflusst wird. Aus letzterem Grund ist die HSC allein heute nicht mehr als Methode der ersten Wahl zu sehen, wenngleich sie eine korrekte Diagnose in $>90 \%$ der Fälle erlaubt. Eine Subtypklassifikation ist nicht möglich.

Die Publikationen, die die Sensitivität und Spezifität der verschiedenen diagnostischen Methoden analysiert haben, wurden in 2 Reviews $[5,18]$ zusammengefasst: Die 2-D-Sonographie, die HSG und die HSC scheinen ungenügend zuverlässig, v. a. wenn sie als einzelne Verfahren verwendet werden. Die zuverlässigste Diagnostik bieten der 3-D-US, die Sonohys- terographie und die LSC kombiniert mit einer HSC (• Tab. 2). Mit diesen Methoden kann eine Sensitivität und Spezifität deutlich $>90 \%$ erreicht werden. Die Genauigkeit der MRT kann noch nicht definitiv beurteilt werden, weshalb auch eine sichere Evaluation dieser Untersuchung bislang nicht möglich ist.

\section{Mögliche renale Fehlbildungen}

Bei ungefähr einem Drittel der Frauen besteht eine Assoziation zwischen uterinen und urogenitalen Fehlbildungen. Diese Tatsache macht eine Kontrolle der Nieren im Falle einer Uterusfehlbildung zwingend notwendig [1]. Je später in der Entwicklung die genitale Fehlbildung auftritt, desto unwahrscheinlicher ist eine renale Fehlbildung. So sind z. B. bei einem Uterus septus die Nieren praktisch immer unauffällig. Eine sonographische Kontrolle ist hier ausreichend. Im Gegensatz dazu treten v. a. beim Uterus unicornis renale Malformationen in fast der Hälfte der Fälle auf. Deshalb sollte beim Nachweis eines Uterus unicornis eine renale Malformation ausgeschlossen und eine MRT-Diagnostik großzügig indiziert werden.

\section{\) Bei Nachweis eines Uterus unicornis ist auch eine renale Malformation auszuschließen}

Zusammengefasst ist ein 3-D-US bei allen Frauen mit habituellen Aborten sinnvoll. Bei Verdacht auf komplizierte Fehlbildungen kann eine MRT indiziert sein, insbesondere wenn die therapeutischen Konsequenzen der Fehlbildung mit der Frau im Detail diskutiert werden müssen, z. B. 
die erhöhte Morbidität bei einer LSC bei besonderen Anomalien wie einem rudimentären Uterushorn. Die Kombination der LSC mit einer HSC sollte Patientinnen vorbehalten bleiben, bei denen eine definitive Beurteilung mit dem 3-D-US nicht möglich ist oder die eine operative Korrektur benötigen.

\section{Therapie der uterinen Fehlbildungen}

Bei Frauen mit rezidivierenden Spontanaborten ist eine operative Therapie bei Nachweis einer Fehlbildung häufig notwendig. Verschiedene Studien haben eine deutliche Verbesserung der Geburtenrate bestätigt [12].

\section{Kanalisationsdefekte: Uterus septus und subseptus}

Uterus septus und subseptus sind die am häufigsten diagnostizierten Fehlbildungen. Sie werden bei etwa 5\% aller Frauen mit habituellen Aborten gefunden. Ihre Behandlung gestaltet sich am einfachsten.

Obwohl randomisierte Studien fehlen [13] und auch ethisch schwierig sind, da Patientinnen mit habituellen Aborten bereits stark unter Stress leiden, bleibt die Therapie der Wahl eine Septumresektion (Metroplastik). Ohne Korrektur wird das Wiederholungsrisiko für einen Abort auf $>50 \%$ geschätzt. Der Mechanismus der Aborte bleibt aber unklar. Lange Zeit wurde die verminderte Vaskularisation im Septumbereich [7] als Hauptfaktor postuliert. Ein neu vorgeschlagener Mechanismus macht nun die reduzierte Expression des „vascular endothelial growth factor" (VEGF) im Fehlbildungsareal verantwortlich. Seit der Einführung der operativen HSC werden Metroplastiken praktisch ausschließlich endoskopisch durch eine transzervikale Resektion mittels einer elektrischen Schlinge oder Nadelelektrode durchgeführt. Die verschiedenen Studien zeigen homogene Resultate mit einer Reduktion der Abortrate auf insgesamt 15\% [9]. Falls eine zuverlässige Diagnostik, d. h. der 3-D-US oder die Sonohysterographie, durchgeführt wurde und der Operateur erfahren ist, kann auf eine gleichzeitige LSC verzichtet werden, obwohl die Genauigkeit und Sicherheit des

Gynäkologische Endokrinologie 2013 · 11:89-94 DOI 10.1007/s10304-012-0517-3

(c) Springer-Verlag Berlin Heidelberg 2013

\section{A. Santi $\cdot$ S. Rohner \\ Anatomische Ursachen bei habituellen Aborten. Diagnostik und Therapie}

\section{Zusammenfassung}

Angeborene Fehlbildungen. Die häufigsten Anomalien des Uterus bei habituellen Aborten sind angeborene Fehlbildungen mit einer Inzidenz von etwa 13\%. Eine frühe, möglichst 3-D-sonographische Diagnostik sollte immer angeboten werden. Der Uterus septus oder subseptus entsteht durch eine fehlende oder mangelnde Resorption des Septums während der Entwicklung. Die Aborte treten meist im späten ersten Trimenon auf. Eine hysteroskopische Septumresektion (Metroplastik) ist angezeigt, da die Geburtenrate auf $>80 \%$ erhöht werden kann. Bei komplizierten Fehlbildungen (Uterus unicornis, bicornis, didelphys) ist eine erweiterte Diagnostik (MRT) sinnvoll, eine operative Korrektur meist aber nicht notwendig, obwohl die Kompli- kationsrate in der Spätschwangerschaft auf einem hohen Niveau verbleibt. Eine begleitende Nierenfehlbildung sollte stets sonographisch oder MR-diagnostisch ausgeschlossen werden.

Erworbene Pathologien. Ob die Diagnose und Behandlung erworbener uteriner Pathologien, z. B. von Myomen, Polypen oder Synechien, das Abortrisiko reduzieren kann, ist noch unklar. Eine operative Behandlung empfiehlt sich dennoch in den meisten Fällen.

\section{Schlüsselwörter}

Habituelle Aborte - Uterine Fehlbildungen . Sonographie - Septumresektion .

Nierenfehlbildung

\section{Anatomical causes of habitual abortion. Diagnostics and treatment}

\section{Abstract}

Congenital malformations. The incidence of congenital uterine malformations in women with recurrent pregnancy loss is $13 \%$. An accurate and objective estimation of the uterus should be offered as soon as possible (particularly using a 3D sonography). The uterus septus or subseptus are the consequence of a defect in the absorption of the uterine septum. Pregnancy loss usually occurs in the late first trimester. A septum resection (metroplasty) by hysteroscopy is indicated, because the birth rate can be improved ( $>80 \%$ ). In complicated malformations (unicornuate uterus, bicornuate, didelphys), advanced diagnostic imaging (MRI) is useful but surgical correction is normally not necessary, al- though the complication rate in late pregnancy remains high. Concomitant renal malformation should always be excluded (by ultrasound or MRI).

Acquired pathologies. Whether the diagnosis and treatment of other uterine diseases (as fibroids, polyps, and adhesions) can reduce the risk of miscarriage is still controversial: surgical treatment is usually recommended.

\section{Keywords}

Miscarriage, recurrent $\cdot$ Uterine malformations - Ultrasonography · Metroplasty $\cdot$ Renal malformation
Eingriffs durch eine LSC erhöht werden können (Verminderung des Perforationsrisikos, Zuverlässigkeit der Fehlbildungsdiagnose).

\section{Uterus arcuatus}

Der Uterus arcuatus ist definiert durch eine geringe Vorwölbung im Fundus uteri. Er wird in etwa $4 \%$ der gesamten Population nachgewiesen und heutzutage als eine nichtrelevante Fehlbildung angesehen, da ihre Häufigkeit bei Frauen mit habituellen Aborten nicht erhöht ist. Aus diesem Grund ist eine Behandlung nicht notwendig.

\section{Defekte der Fusion}

Der Uterus unicornis ist eine Fehlbildung mit der regelrechten Entwicklung von nur einem Horn (• Abb. 3). In rund 70\% der Fälle ist ein Resthorn vorhanden. Dieses ist aber meist nicht mit dem anderen Horn verbunden (nichtkommunizierendes Resthorn in etwa 75\% der Fälle). Eine 


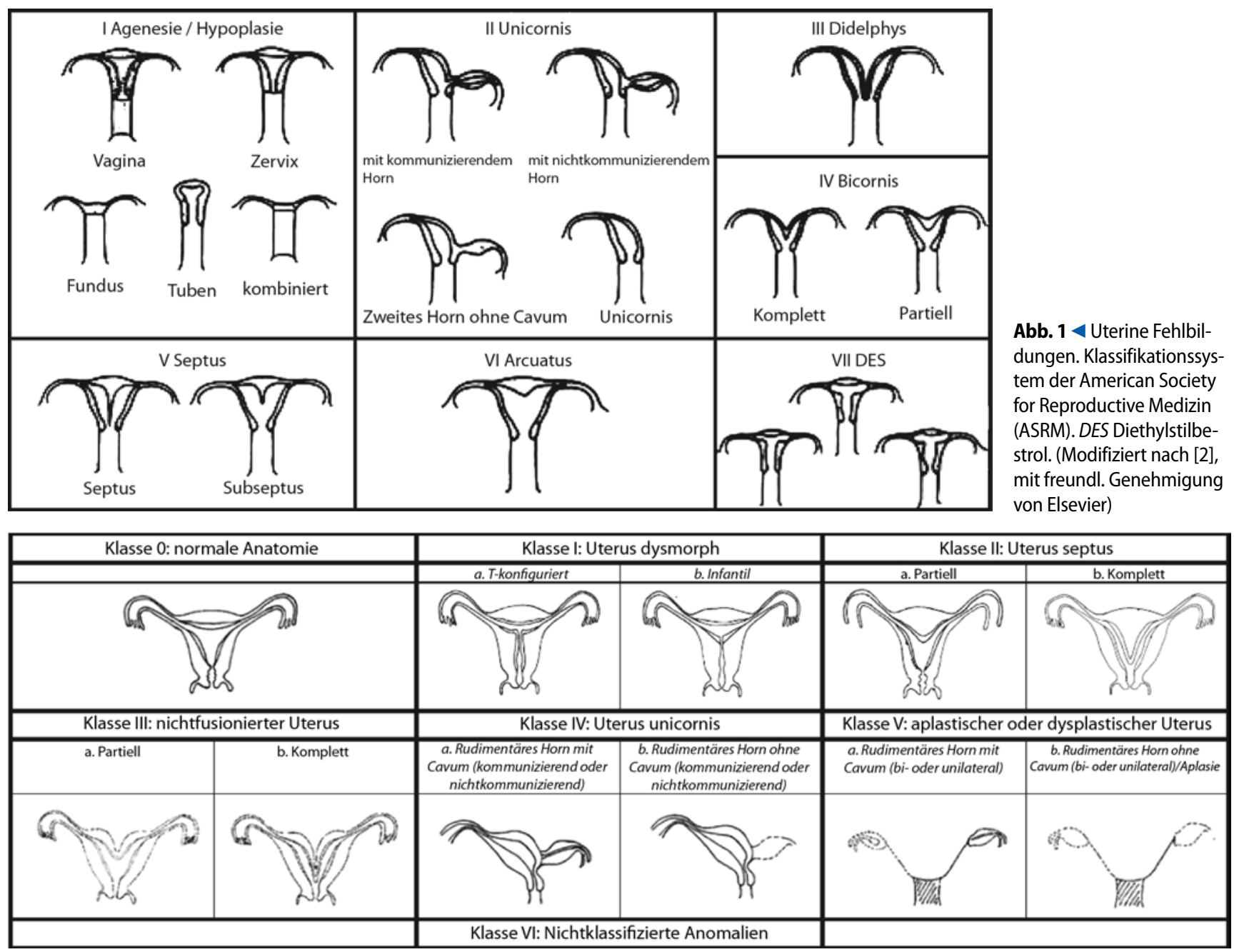

Abb. $2 \Delta$ Klassifikation uteriner Fehlbildungen. Neuer Vorschlag der European Society of Human Reproduction and Embryology (ESHRE) und der European Society for Gynaecological Endoscopy (ESGE). (Adaptiert nach [11])

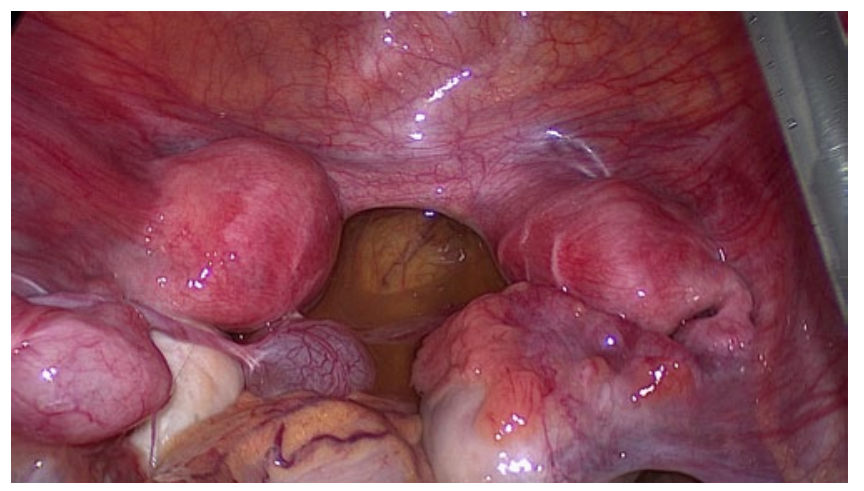

Abb. $3<$ Laparoskopisches Bild eines Uterus unicornis mit rudimentärem nichtkommunizierendem Horn. Links Uterus unicornis mit Cavum und normaler Verbindung zur Vagina; rechts rudimentärer Uterusteil ohne vaginale Verbindung

Therapie ist deshalb nur möglich und notwendig, falls die Patientin symptomatisch wird, z. B. durch eine ausgeprägte Dysmenorrhö bei Hämatometra im rudimentären Horn. Das Abortrisiko ist in dieser Konstellation leider, wie auch die Rezidivrate, hoch [15].
Der Uterus bicornis ist definiert als eine Zervix mit 2 Corpora. Hier sind klinisch die geburtshilflichen Komplikationen von größerer Bedeutung, da sich häufig eine Zervixinsuffizienz mit Frühgeburtlichkeit als Konsequenz ausbildet. $\mathrm{Ob}$ die Häufigkeit habitueller Aborte ebenfalls erhöht ist, bleibt unklar. Ein operati- ver Eingriff ist nur in speziellen Situationen sinnvoll, z. B. eine Cerclage bei einer Zervixinsuffizienz.

Beim Uterus didelphys finden wir 2 separate Uteri (2 Corpora und 2 Zervices), die nicht kommunizieren. Diese relativ seltene Missbildung ist häufig mit einem vaginalen Septum kombiniert. Heutzutage ist eine vaginale Korrektur nur im Falle einer Dyspareunie angezeigt, eine operative Korrektur der Uteri ist in der Regel nicht notwendig. Wie auch in anderen Fällen einer gestörten Fusion der MüllerGänge treten Komplikationen v. a. in der Spätschwangerschaft deutlich vermehrt auf [19].

\section{Seltene uterine Fehlbildungen}

Der hypoplastische Uterus mit T-Konfiguration („T-shaped“) ist heute eine 


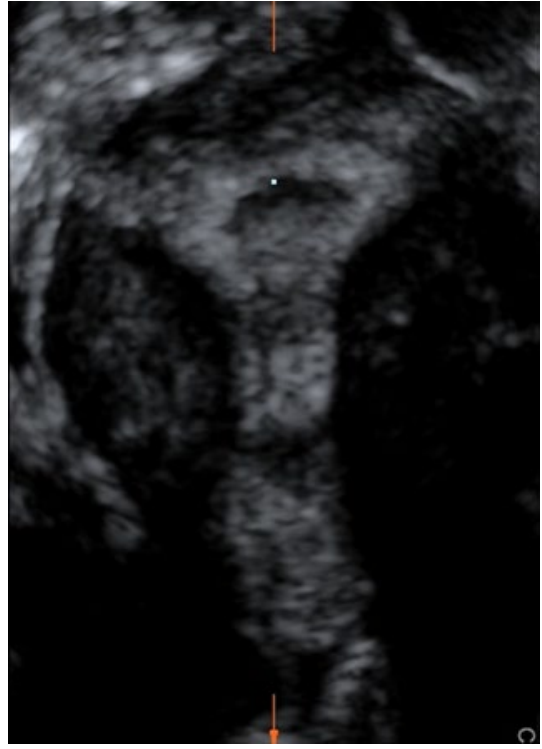

Abb. 4 \ 3-D-sonographisches Bild eines Uterus mit T-Konfiguration (,T-shaped ${ }^{\prime \prime}$ ). Das Endometrium ist sehr schmal und bildet eine typische T-Form

Rarität unter den uterinen Fehlbildungen $(<1 \%)$. Sie kommt sowohl bei sterilen Frauen als auch bei habituellen Aborten vor (• Abb.4). Diese Anomalie wurde v. a. bei jenen Frauen diagnostiziert, deren Mütter während der Schwangerschaft Diethylstilbestrol (DES) eingenommen hatten. Eine Arbeit von 2011 [8] hat die Metroplastik als positiv beurteilt. Die Geburtenrate wurde deutlich verbessert.

\section{Erworbene uterine Pathologien}

Die Bedeutung und Konsequenzen von uterinen Anomalien wie Polypen, Myomen und Synechien bei infertilen Frauen werden seit Jahren kontrovers diskutiert. Derzeit fehlen noch klare Daten, die aufzeigen, dass eine operative Korrektur dieser Befunde eine signifikante Verbesserung der Geburtenrate bei Frauen mit rezidivierenden Aborten bewirken kann. Andererseits ist v. a. eine spezifische Behandlung intrakavitärer Pathologien wie submuköser Myome und Polypen meist sinnvoll, da die Operation mit einem geringen Risiko und Aufwand verbunden ist [18].

\section{Intrauterine Synechien}

Das sog. Asherman-Syndrom ist eine mögliche Konsequenz intrakavitärer
Uterusoperationen. Es tritt nicht zuletzt nach Abrasio auf. Die Häufigkeit variiert zwischen $13 \%$ nach einer Abrasio und fast 50\% nach einer hysteroskopischen Enukleation mehrerer submuköser Myome [14]. Ebenfalls sehr anfällig für die Entwicklung intrauteriner Adhäsionen scheint die Nachkürettage 2-4 Wochen post partum zu sein. Die beste Therapie ist hier die Prävention, da leider eindeutige Daten fehlen, die die Effizienz der hysteroskopischen Synechienlösung bestätigen könnten.

\section{Fazit für die Praxis}

- Anatomische Ursachen sollten bei habituellen Aborten immer ausgeschlossen werden, da eine uterine Fehlbildung in etwa 15\% der Fälle als möglicher Kofaktor vorhanden ist.

- Der erste diagnostische Schritt ist der 2-D-US. Empfohlen ist aber auch eine 3-D-Beurteilung, da sie die Zuverlässigkeit der Diagnose deutlich erhöht.

- Die HSG hat in der Diagnostik uteriner Fehlbildungen bei habituellen Aborten keinen Stellenwert mehr.

- Die MRT sollte lediglich bei komplizierten Fehlbildungen veranlasst werden, nicht zuletzt wegen der hohen Kosten.

- Die Durchführung einer diagnostischen LSC mit HSC bleibt jenen Frauen vorbehalten, bei denen die nichtinvasive Abklärung nicht eindeutig war.

- Die häufigsten intrauterinen Anomalien sind Septen im Cavum uteri (Uterus septus/subseptus). Hier ist eine chirurgische Korrektur mithilfe der hysteroskopischen Metroplastik sinnvoll und rasch zu empfehlen, da sich die Geburtenrate in der Folge deutlich erhöht.

- Inwieweit die operative Behandlung erworbener intrauteriner Pathologien positive Effekte hat, wird weiterhin kontrovers diskutiert. Eine Therapie scheint aber auch in diesen Fällen angezeigt zu sein.

\section{Korrespondenzadresse}

\section{Dr. A. Santi}

Kinderwunschzentrum,

Universitätsklinik für Frauenheilkunde,

Inselspital Bern

Effingerstr. 102, 3010 Bern

Schweiz

alessandro.santi@insel.ch

Interessenkonflikt. Der korrespondierende Autor gibt für sich und seine Koautorin an, dass kein Interessenkonflikt besteht.

\section{Literatur}

1. Acién P, Acién M, Sánchez-Ferrer M (2004) Complex malformations of the female genital tract. New types and revision of classification. Hum Reprod 19:2377-2384

2. American Fertility Society (1988) The American Fertility Society classifications of adnexal adhesions, distal tubal occlusion, tubal occlusion secondary to tubal ligation, tubal pregnancies, mullerian anomalies and intrauterine adhesions. Fertil Steril 49:944-955

3. Bermejo C, Martínez Ten P, Cantarero R et al (2010) Three-dimensional ultrasound in the diagnosis of Mullerian duct anomalies and concordance with magnetic resonance imaging. Ultrasound Obstet Gynecol 35:593-601

4. Caliskan E, Ozkan S, Cakiroglu Y et al (2010) Diagnostic accuracy of real-time 3D sonography in the diagnosis of congenital Mullerian anomalies in high-risk patients with respect to the phase of the menstrual cycle. J Clin Ultrasound 38:123-127

5. Chan YY, Jayaprakasan K, Zamora J et al (2011) The prevalence of congenital uterine anomalies in unselected and high-risk populations: a systematic review. Hum Reprod Update 17:761-771

6. Faivre E, Fernandez H, Deffieux X et al (2012) Accuracy of three-dimensional ultrasonography in differential diagnosis of septate and bicornuate uterus compared with office hysteroscopy and pelvic magnetic resonance imaging. J Minim Invasive Gynecol 19:101-106

7. Fayez JA (1986) Comparison between abdominal and hysteroscopic metroplasty. Obstet Gynecol 68:399-403

8. Fernandez H, Garbin O, Castaigne V et al (2011) Surgical approach to and reproductive outcome after surgical correction of a T-shaped uterus. Hum Reprod 26:1730-1734

9. Grimbizis GF, Camus M, Tarlatzis BC et al (2001) Clinical implications of uterine malformations and hysteroscopic treatment results. Hum Reprod Update 7:161-174

10. Grimbizis GF, Tsolakidis D, Mikos T et al (2010) A prospective comparison of transvaginal ultrasound, saline infusion sonohysterography, and diagnostic hysteroscopy in the evaluation of endometrial pathology. Fertil Steril 94:2720-2725

11. Grimbizis GF, Campo R (2012) Clinical approach for the classification of congenital uterine malformations. Gynecol Surg 9:119-129

12. Homer HA, Li TC, Cooke ID (2000) The septate uterus: a review of management and reproductive outcome. Fertil Steril 73:1-14 
13. Kowalik CR, Goddijn M, Emanuel MH et al (2011) Metroplasty versus expectant management for women with recurrent miscarriage and a septate uterus. Cochrane Database Syst Rev 6:CD008576

14. March CM (2011) Management of Asherman's syndrome. Reprod Biomed Online 23:63-76

15. Nahum GG (2002) Rudimentary uterine horn pregnancy. The 20th-century worldwide experience of 588 cases. J Reprod Med 47:151-163

16. Olpin JD, Heilbrun M (2009) Imaging of Mullerian duct anomalies. Clin Obstet Gynecol 52:40-56

17. Renbaum L, Ufberg D, Sammel M et al (2002) Reliability of clinicians versus radiologists for detecting abnormalities on hysterosalpingogram films. Fertil Steril 78:614-618

18. Saravelos SH, Cocksedge KA, Li TC (2008) Prevalence and diagnosis of congenital uterine anomalies in women with reproductive failure: a critical appraisal. Hum Reprod Update 14:415-429

19. Sefrioui O, Azyez M, Babahabib A et al (2004) Pregnancy in rudimentary uterine horn: diagnostic and therapeutic difficulties. Gynecol Obstet Fertil 32:308-310

\section{Medizinstudenten üben künftig Patientengespräche}

In Zukunft sollen angehende Ärzte Patientengespräche schon ab dem ersten Semester üben. Das sieht ein deutschlandweites Kommunikationscurriculum für Medizinstudenten vor, das derzeit erarbeitet wird.

Studien zeigen: Wenn Arzt und Patient zwar dieselbe Sprache sprechen, einander aber nicht verstehen, kann dies zu falschen Diagnosen und Fehlbehandlungen führen. Ein "guter Draht" zwischen Arzt und Patient fördert hingegen die Therapietreue. Denn nur wenn der Patient sich verstanden fühlt und seinem Arzt vertraut, kooperiert er auch bei der Therapie.

Vertreter aller deutschen medizinischen

Fakultäten tragen jetzt die besten Übungen und Trainingsmöglichkeiten zusammen, um gemeinsam ein Kommunikationscurriculum für alle Studierenden zu entwickeln. Die Patientengespräche sollen Studenten ihr ganzes Studium hindurch begleiten, wobei die Gesprächssituationen im Laufe der Zeit immer komplexer werden. Das Spektrum reicht von diagnostischen Übungen über Gespräche mit Eltern, deren Kinder behandelt werden, bis hin zum Überbringen schlechter Nachrichten und Gesprächen mit nicht-therapietreuen Patienten.

Im Herbst 2014 soll das Kommunikationscurriculum verabschiedet werden. Das kommt dann nicht nur den Patienten zugute, sondern auch den Ärzten. Denn wenn die Kommunikation mit den Patienten "rund läuft", entwickeln sie seltener ein Burnout-Syndrom.

\section{Quelle:}

Arbeitsgemeinschaft der Wissenschaftlichen Medizinischen Fachgesellschaften (AWMF), www.awmf.org

\section{Schmerz-Appell}

Mit einem Appell an die Gesundheitspolitik möchte die Deutsche Schmerzgesellschaft bessere Rahmenbedingungen für Versorgungsforschung sowie den Ausbau von Schmerzregistern in Deutschland erreichen. Sie setzt sich damit nicht nur für eine verbesserte Patientenversorgung ein, auch gute Verträge und eine faire Honorierung werden angestrebt. Zu diesem Zwecke ruft die Schmerzgesellschaft zu einer großen Unterschriftenaktion auf. Sie als Schmerztherapeut oder Schmerzforscher sind nun gefragt. Es zählen Ihre Meinung und Ihre Unterschrift. Auch Ihre Kollegen können den Aufruf gerne verteilen und unterschreiben. Die Unterschriften werden bis Herbst/ Winter 2013 gesammelt. Nach der nächsten Bundestagswahl soll der Appell der Bundesregierung übergeben werden.

Den vollständigen Appell sowie das Unterschriftenformular finden Sie Internet unter www.dgss.org/versorgung/schmerz-appell.

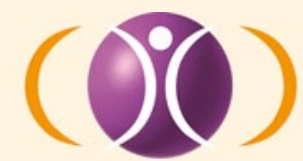

Deutsche Schmerzgesellschaft e.V. Sektion der International Association for the Study of Poin (uSP)

Ihre ausgefüllte Unterschriftenliste senden Sie bitte an die:

Bundesgeschäftsstelle der Deutschen Schmerzgesellschaft e. V., Alt-Moabit 101b, 10559 Berlin, per Fax: 030-39409689-9 oder per Mail an info@dgss.org. 